\title{
Electron-Cloud Effects in Transport Lines of a Normal Conducting Linear Collider*
}

\author{
Juhao $\mathrm{Wu}^{\dagger}$, T.O. Raubenheimer, M.T.F. Pivi, and A. Seryi \\ SLAC, Menlo Park, CA 94025, USA
}

\section{Abstract}

In the transport lines of a normal conducting linear collider, the long positron bunch train can generate an electron cloud which can then amplify intra-train offsets. This is a transient effect which is similar to the electron-cloud driven coupled bunch instabilities in a positron storage ring. In this paper, we study this phenomenon analytically. Some criteria on the critical cloud density with respect to given collider parameters are discussed.

\section{Introduction}

In this paper, we study multi-bunch electron-cloud effect within linear theory. The coordinate system is shown in Fig. 1. Physically, the electron cloud should overlap with the positron bunch train. However, to show the definition of the coordinates, we give the positron bunch train with some vertical lift in the plot. The physics problem is the following, assume the beam line begins at $s=0$ at $t=0$, we want to know how the bunch train is disturbed due to beam-electron-cloud interaction. The positive $s$ direction is to the right, while the internal positron bunch train coordinate $z$ is pointing to the left.

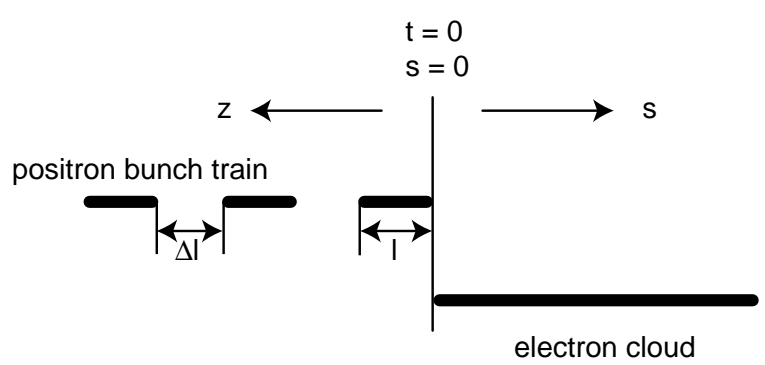

Figure 1: Schematic of the coordinate system for the positron bunch train and the electron cloud. The definition of the notations: $s, z, l$, and $\Delta l$ are also shown.

We here assume that the bunches in the positron bunch train have a separation of $\Delta l$ and a bunch length of $l$. For the US Warm [1], $\Delta l \approx 0.42 \mathrm{~m}$ and $l \approx 0.38 \mathrm{~mm}$, and it is a good approximation to regard it as a $\delta$-bunch train. In the US Cold design, the large bunch separation of $\sim 100 \mathrm{~m}$ prevents the electron-cloud from building up.

\footnotetext{
* Work supported by the U.S. Department of Energy under Contract No. DE-AC02-76SF00515.

$\dagger$ jhwu@SLAC.Stanford.EDU
}

\begin{tabular}{ccc}
\hline \hline & US Cold & US Warm \\
\hline Energy per beam $(\mathrm{GeV})$ & 250 & 250 \\
$N\left(10^{10}\right)$ & 2.0 & 0.75 \\
$\sigma_{x}(\mu \mathrm{m})$ & 0.543 & 0.243 \\
$\sigma_{x^{\prime}}(\mu \mathrm{rad})$ & 36 & 30 \\
$\sigma_{y}(\mathrm{~nm})$ & 5.7 & 3.0 \\
$\sigma_{y^{\prime}}(\mu \mathrm{rad})$ & 14 & 27 \\
$\sigma_{z}(\mathrm{~mm})$ & 0.3 & 0.11 \\
$\sigma_{\delta}(\%)$ & 0.1 & 0.3 \\
$N_{b}$ & 2820 & 192 \\
$\Delta t(\mathrm{~ns})$ & 337 & 1.4 \\
\hline$n_{e}$ & $1.58 \times 10^{11}$ & $1.42 \times 10^{13}$ \\
$\varepsilon_{b}\left(\mathrm{~m}^{-2}\right)$ & $1.90 \times 10^{-9}$ & $1.72 \times 10^{-7}$ \\
\hline \hline
\end{tabular}

Table 1: Summary of the parameters for the US Cold and US Warm [1].

\section{Multi-bunch effect: $\delta$-bunch train beam breakup in a building up electron cloud}

Let us now study a $\delta$-bunch train. The method is similar to that in Ref. [2]. As above, $y_{j}(s)$ is the transverse coordinate of the $j$-th bunch when it passes the point $s$. We approximate the focusing structure by a smooth focusing function $k_{\beta}=2 \pi /$ (local betatron wave length). We assume every bunch has the same charge, then the equation of motion for the $j$-th positron bunch is

$$
\frac{d^{2} y_{j}(s)}{d s^{2}}+k_{\beta}^{2} y_{j}(s)=\varepsilon_{b} \sum_{k=0}^{j} w\left(z_{j}-z_{k}\right) F\left(z_{k}\right) y_{k}(s),
$$

where $\varepsilon_{b}=\varepsilon z_{b}$ with $z_{b}$ being the bunch separation; $z_{j}=$ $j z_{b}$ the distance of the $j$-th bunch from the first (zero) bunch; $w(z)$ is the transverse wake due to the electroncloud. Causality requires that $w(z)=0$ for $z<0$. To approximate the buildup process, the current function is written

$$
F(z)=1-e^{-\alpha z} .
$$

The electron-cloud buildup, $\alpha$, will depend on the secondary electron yield, vacuum chamber geometry, etc.

Equation (1) can be solved via Laplace transform, and yields the following solution

$y_{j}(s)=\sum_{n=0}^{\infty} \varepsilon_{b}^{n}\left[y_{0} h_{n}\left(z_{j}\right) j_{n}\left(k_{\beta}, s\right)+y_{0}^{\prime} g_{n}\left(z_{j}\right) i_{n}\left(k_{\beta}, s\right)\right]$,

and

$$
y_{j}^{\prime}(s)=y_{0}^{\prime} \sum_{n=0}^{\infty} \varepsilon_{b}^{n} g_{n}\left(z_{j}\right) j_{n}\left(k_{\beta}, s\right)+y_{0}\left\{-k_{\beta}^{2} h_{0}\left(z_{j}\right) i_{0}\left(k_{\beta}, s\right)\right.
$$




$$
\left.+\sum_{n=1}^{\infty} \varepsilon_{b}^{n} h_{n}\left(z_{j}\right)\left[i_{n-1}\left(k_{\beta}, s\right)-k_{\beta}^{2} i_{n}\left(k_{\beta}, s\right)\right]\right\}
$$

with

$$
\begin{gathered}
i_{n}\left(k_{\beta}, s\right)=\frac{1}{n !}\left(\frac{s}{2 k_{\beta}}\right)^{n} \frac{1}{k_{\beta}} \sqrt{\frac{\pi k_{\beta} s}{2}} J_{n+1 / 2}\left(k_{\beta} s\right), \\
j_{n}\left(k_{\beta}, s\right)=\frac{s}{2 n} i_{n-1}\left(k_{\beta}, s\right),
\end{gathered}
$$

and

$$
\left\{\begin{array}{l}
g_{n+1}\left(z_{j}\right) \\
h_{n+1}\left(z_{j}\right)
\end{array}\right\}=\sum_{k=0}^{j} w\left(z_{j}-z_{k}\right) F\left(z_{k}\right)\left\{\begin{array}{l}
g_{n}\left(z_{k}\right) \\
h_{n}\left(z_{k}\right)
\end{array}\right\} .
$$

The initial conditions are introduced as

$$
y_{0}^{\prime} g_{0}\left(z_{j}\right)=y_{0}^{\prime}\left(z_{j}\right)=\left.\frac{d y_{j}(s)}{d s}\right|_{s=0},
$$

and

$$
y_{0} h_{0}\left(z_{j}\right)=y_{0}\left(z_{j}\right)=y_{j}(0) .
$$

The wake due to the electron-cloud can be approximated as a single deflecting mode with loss, i.e., [3, 4]

$$
w(z)=e^{-\eta z} \sin \left(k_{e} z\right) .
$$

Notice that, we have put the amplitude in the parameter $\varepsilon$. In very loose speaking, for the practical system, we are only interested in $\varepsilon_{b}$ being small. Otherwise, the system will not work. Hence, in the following, we will work out the first-order solution, and see how the electron buildup differs from that of a steady state.

Assuming $h_{0}\left(z_{j}\right)=g_{0}\left(z_{j}\right)=1$, we find

$$
\begin{aligned}
& h_{1}\left(\eta, z_{b}, \alpha, k_{e}, z_{j}\right)=g_{1}\left(\eta, z_{b}, \alpha, k_{e}, z_{j}\right) \\
= & h_{1}^{s s}\left(\eta, z_{b}, k_{e}, z_{j}\right)-e^{-j \alpha z_{b}} h_{1}^{s s}\left(\eta-\alpha, z_{b}, k_{e}, z_{j}\right),
\end{aligned}
$$

where

$$
\begin{aligned}
& h_{1}^{s s}\left(\eta, z_{b}, k_{e}, z_{j}\right)=g_{1}^{s s}\left(\eta, z_{b}, k_{e}, z_{j}\right) \\
= & \frac{e^{-\eta z_{b}}}{1-2 e^{-\eta z_{b}} \cos \left(k_{e} z_{b}\right)+e^{-2 \eta z_{b}}} \\
\times & {\left[\sin \left(k_{e} z_{b}\right)\left\{1-e^{-\eta(j+1) z_{b}} \cos \left[k_{e}(j+1) z_{b}\right]\right\}\right.} \\
- & \left.e^{-\eta j z_{b}} \sin \left[k_{e}(j+1) z_{b}\right]\left\{1-e^{-\eta z_{b}} \cos \left(k_{e} z_{b}\right)\right\}\right],
\end{aligned}
$$

are for the steady state, i.e., for $F(z)=1$. So, the buildup process introduces a term with a modified quality factor $Q$ relates to $\eta$ by $Q=k_{e} /(2 \eta)$. Interestingly, if $\eta=\alpha$, then the buildup will compensate the loss. Also, this term is transient in nature, and decays very soon, once $j \alpha z_{b}$, or more precisely, $[(j-1) \alpha+\eta] z_{b}$ gets reasonably large.

The wake amplitude is defined as $[3,4] \varepsilon=k_{p}^{2} k_{e}$, with

$$
k_{p}^{2}=\frac{n_{e} r_{e}}{\gamma}=\frac{N_{p} r_{e}}{\pi b^{2} \gamma z_{b}} \quad \text { and } \quad k_{e} \approx \frac{2 \pi}{3 z_{b}}
$$

where $N_{p}$ is the positron population per bunch, and $b$ is the vacuum pipe radius. In getting the above focusing strength in Eq. (13), we have used the neutralization condition, i.e., $n_{e}=N_{p} /\left(\pi b^{2} z_{b}\right)$. The corresponding $\varepsilon_{b}$ is given in Table 1. In getting the threshold, then we will treat $n_{e}$ as a free parameter. We want to point out here that, there are possible resonance phenomena when $k_{e} z_{b}$ takes "appropriate" values. However, such resonance may have a very narrow bandwidth, hence may not be able to build up coherently. In this paper, we will treat the beam-electron-cloud interaction as perturbation, hence we are in the regime where $h_{1}\left(z_{j}\right) \sim 1$, especially when we study the threshold.

Explicitly, we have

$$
\begin{aligned}
y_{j}(s) & =y_{0} \cos \left(k_{\beta} s\right)+y_{0}^{\prime} \frac{\sin \left(k_{\beta} s\right)}{k_{\beta}} \\
& +s \frac{\varepsilon_{b} h_{1}\left(z_{j}\right)}{2 k_{\beta}}\left\{y_{0} \sin \left(k_{\beta} s\right)\right. \\
& \left.-y_{0}^{\prime}\left[\frac{\cos \left(k_{\beta} s\right)}{k_{\beta}}-\frac{\sin \left(k_{\beta} s\right)}{k_{\beta}^{2} s}\right]\right\}+\mathcal{O}\left(\varepsilon_{b}^{2}\right)
\end{aligned}
$$

and

$$
\begin{aligned}
y_{j}^{\prime}(s) & =-y_{0} k_{\beta} \sin \left(k_{\beta} s\right)+y_{0}^{\prime} \cos \left(k_{\beta} s\right) \\
& +s \frac{\varepsilon_{b} h_{1}\left(z_{j}\right)}{2}\left\{y_{0}\left[\cos \left(k_{\beta} s\right)+\frac{\sin \left(k_{\beta} s\right)}{k_{\beta} s}\right]\right. \\
& \left.+y_{0}^{\prime} \frac{\sin \left(k_{\beta} s\right)}{k_{\beta}}\right\}+\mathcal{O}\left(\varepsilon_{b}^{2}\right) .
\end{aligned}
$$

Given these, one then can look at the secular behavior by only keeping terms proportional to $s$ of the perturbation terms. Also, one can deal with realistic beam delivery system, by constructing a linear transport matrix for each element using Eqs. (14) and (15).

At this stage, maybe it is worth to mention that $h_{1}\left(z_{j}\right)$ has the following expression for different regime:

$$
\begin{aligned}
& h_{1}\left(\eta, z_{b}, \alpha, k_{e}, z_{j}\right)=g_{1}\left(\eta, z_{b}, \alpha, k_{e}, z_{j}\right) \\
= & \left\{\begin{array}{ll}
j \alpha z_{b} h_{1}^{s s}\left(\eta, z_{b}, k_{e}, z_{j}\right)+\alpha \frac{\partial h_{1}^{s s}\left(\eta, z_{b}, k_{e}, z_{j}\right)}{\partial \eta} & \text { for } \alpha \ll 1 \\
h_{1}^{s s}\left(\eta, z_{b}, k_{e}, z_{j}\right)-e^{-j \alpha z_{b}} h_{1}^{s s}\left(\eta-\alpha, z_{b}, k_{e}, z_{j}\right) \\
h_{1}^{s s}\left(\eta, z_{b}, k_{e}, z_{j}\right) & \text { for } \alpha \gg 1
\end{array},\right.
\end{aligned}
$$

Namely, if the electron-cloud buildup process is very slow in the interested time duration, then we are in the linear growth regime, i.e., $\alpha \ll 1$. While in the other limit, i.e., the electron-cloud builds up very soon, then we are in the steady state regime, i.e., $\alpha \gg 1$.

\section{Results}

Now, let us look at the US Warm case, we are interested in the final focusing system. In the final transformer, $\beta \approx 50 \mathrm{~km}$, the total length is about $300 \mathrm{~m}$, and vacuum pipe radius is taken to be $b=2 \mathrm{~cm}$. According to simulation, $Q \approx 5$, i.e., $\eta \approx k_{e} / 10$. In this paper, we study two possible build up speed. In the first case, we assume around the 20-th, the electron-loud reaches steady-state. This gives $\alpha \approx 1 /\left(5 z_{b}\right) \approx k_{e} /(10 \pi / 3)$. We are in the regime of $\alpha \sim \eta<k_{e}$. The second, we assume that it needs 100 
bunches to reach steady-state, then $\alpha \approx k_{e} /(50 \pi / 3)$. We are in the regime of $\alpha<\eta<k_{e}$.

Since $k_{\beta} s \leq 0.006$, we expand Eq. (14) as

$$
y_{j}(s) \approx y_{0}+y_{0}^{\prime} s+\frac{\varepsilon_{b} h_{1}\left(z_{j}\right)}{2} y_{0} s^{2}+\frac{\varepsilon_{b} h_{1}\left(z_{j}\right)}{6} y_{0}^{\prime} s^{3} .
$$

Similarly, according to Eq. (15), we have

$$
y_{j}^{\prime}(s) \approx y_{0}^{\prime}+\varepsilon_{b} h_{1}\left(z_{j}\right) y_{0} s+\frac{\varepsilon_{b} h_{1}\left(z_{j}\right)}{2} y_{0}^{\prime} s^{2} .
$$

Now let us study how this may affect the final beam size at the Intersection Point (IP). The main effect comes from the angular kick in the final transformer. This angular kick will affect the collision location or the beam size at IP. According to Eq. (18), the angular kick is roughly

$$
\Delta y^{\prime}=\varepsilon_{b} h_{1}\left(z_{j}\right) y_{0} s,
$$

where one may take the jitter amplitude $y_{0}=\mathcal{N} \sigma_{y}$ with $\mathcal{N}$ being a number.

At IP, the motion is denoted as

$$
\Delta y^{*}=\int \sqrt{\beta^{*} \beta(s)} \sin \left[\varphi^{*}-\varphi(s)\right] \theta(s) d s,
$$

where $\beta^{*}$ and $\varphi^{*}$ are the betatron number and phase at IP; and $\theta(s)$ is the angular kick per unit length which is equal to $\theta=\varepsilon_{b} h_{1}\left(z_{j}\right) y_{0}$ according to Eq. (19). Hence, for the final transformer, i.e., the $300 \mathrm{~m}$ upstream of the final doublet, Eq. (20) is simplified as

$$
\Delta y^{*} \sim \sqrt{\beta^{*} \beta} \varepsilon_{b} h_{1}\left(z_{j}\right) y_{0} s .
$$

This yields

$$
\frac{\Delta y^{*}}{\sigma_{y}^{*}}=\mathcal{N} \beta s \varepsilon_{b} h_{1}\left(z_{j}\right) .
$$

As a quick estimate, we take $\mathcal{N}=1$, i.e., $1 \sigma_{y}$ initial jitter, and $h_{1}\left(z_{j}\right) \sim 1$, then given the electron-cloud density of the neutralization, we would have $\Delta y^{*} / \sigma_{y}^{*} \sim 2.6$, which indicates that electron-cloud should be an issue.

To be more precise, we take $\mathcal{N}=1$, and $h_{1}\left(z_{j}\right)$ from Eq. (11), and show the results in Fig. 2, where the red solid curve stands for the steady-state result; the blue dotted curve is for fast buildup, i.e., the buildup is within 20 bunches; the green dashed curve is for slow buildup within 100 bunches.

Given these parameters, then it shows that to keep $\Delta y^{*} / \sigma_{y}^{*}<10 \%$, we need to keep $n_{e}<2 \times 10^{12}\left(\mathrm{~m}^{-3}\right)$. Some details are shown in Fig. 3. Since we choose $\mathcal{N}=1$, the results should be scaled accordingly with $\mathcal{N}$.

\section{Discussion}

In this paper, we study the multi-bunch effect due to the beam-electron-cloud interaction. The results for $k_{e} z_{b}=$ $2 \pi / 3$ show that such effect would be an issue, if the system reaches neutralization. The threshold would be around $n_{e} \sim 10^{12}\left(\mathrm{~m}^{-3}\right)$ for $\Delta y^{*} / \sigma_{y}^{*}<10 \%$. More detailed study is needed to explore the importance of $k_{e} z_{b}$ including possible resonance phenomena.

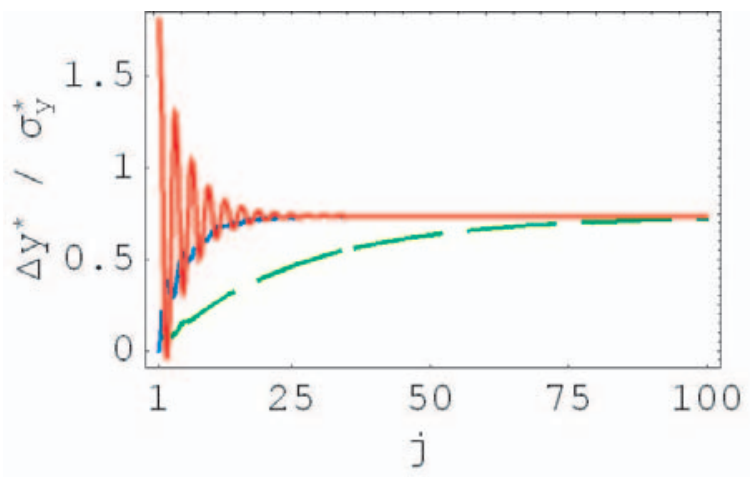

Figure 2: Effect of the beam-electron-cloud interaction for different bunches in the train. The vertical axis is for $\Delta y^{*} / \sigma_{y}^{*}$ at $s=300 \mathrm{~m}$, and the horizontal axis is the bunch index.

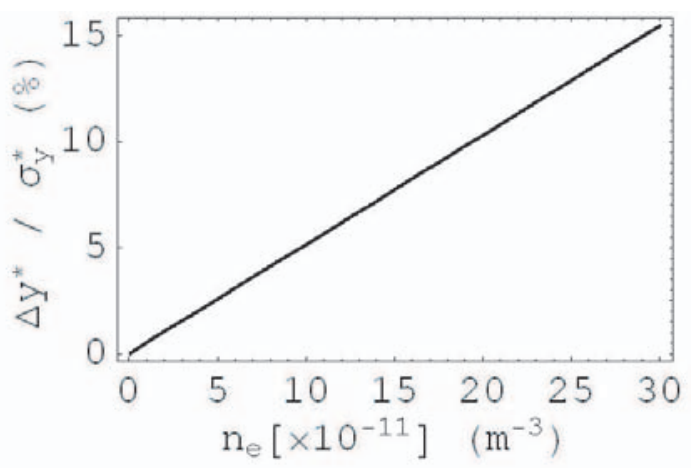

Figure 3: Effect of the beam-electron-cloud interaction for different electron-cloud equilibrium density $n_{e}\left(\mathrm{~m}^{-3}\right)$. The vertical axis is for $\Delta y^{*} / \sigma_{y}^{*}$ at $s=300 \mathrm{~m}$, and the horizontal axis is the electron density.

\section{REFERENCES}

[1] http://www.slac.stanford.edu/xorg/accelops/.

[2] J.R. Delayen, Phys. Rev. ST Accel. Beams 6, 084402 (2003).

[3] K. Ohmi, F. Zimmermann, and E. Perevedentsev, Phys. Rev. E 65, 016502 (2002).

[4] S. Heifets, SLAC-PUB-9105, January 2002. 